\title{
"¿Diferencias encubiertas entre grupos? Efecto del método analítico sobre las conclusiones", en relación al artículo"Medidas directas e indirectas de las funciones ejecutivas en niños con trastorno de espectro autista."
}

\author{
Response to letter to the editor "Hidden differences \\ between groups? Effect of analytical method on the \\ conclusions" in relation to the article "Direct and indirect \\ measures of executive functions in children with autism \\ spectrum disorder".
}

Estimado Sr. Editor:

Agradecemos el espacio para dar respuesta a la carta " ¿Diferencias encubiertas entre grupos? Efecto del método analítico sobre las conclusiones" así como a sus autores, por las observaciones oportunas sobre la importancia de reportar la magnitud del efecto (ME) que enriquecen el análisis de los resultados de nuestro trabajo titulado: "Medidas directas e indirectas de las funciones ejecutivas en niños con trastorno de espectro autista."

La comparación de las funciones ejecutivas (FE) entre niños con y sin trastorno de espectro autista (TEA), muestra resultados diferentes. Por una parte, la medición indirecta (MI) de las FE, por ejemplo, mediante el reporte de los padres sobre la conducta de sus hijos, parece ser más consistente a favor de una alteración ejecutiva en el grupo con TEA. ${ }^{1-4}$ En tanto que la medición directa (MD) de las FE, con pruebas de ejecución, los resultados han sido inconsistentes. ${ }^{5}$

En nuestro estudio sobre las FE en niños con y $\sin \mathrm{TEA},{ }^{6}$ los resultados mostraron diferencias en la mayoría de las MI (prueba BRIEF), pero no en las MD (prueba ENFEN). EI marco analítico de nuestros datos, sobre los cuales se basaron nuestras conclusiones, se realizó en condiciones 
de una muestra pequeña $(n=14)$ y que no se ajustó a una distribución normal (Shapiro-Wilk). ${ }^{7}$ Empleando el estadístico $U$ de Mann-Witney, ${ }^{8}$ con un nivel de significancia de $p \leq 0.05$.

Sin embargo, reconocemos que la omisión de la magnitud del efecto (ME), restringe las conclusiones realizadas únicamente sobre la base de prueba de significancia de hipótesis nula (PSHN). En este sentido, consideramos oportuno emplear el valor de la $M E$, por sus aportaciones a nuestras conclusiones, como es señalado en la carta al editor titulada: ¿Diferencias encubiertas entre grupos? Efecto del método analítico sobre las conclusiones.

Incluir la $M E$, permitiría: 1) valorar de manera más realista la relevancia de las diferencias entre los grupos, dentro del marco teórico y práctico que contextualizan la investigación, 2) analizar los datos con muestras pequeñas $(\mathrm{n}<30)$ y 3 ) integrar los resultados con los datos de diferentes investigaciones, en estudios posteriores de metanálisis. Para el análisis de la ME ( $d$ de Cohen), ${ }^{9}$ se consideraron los siguientes puntos de corte propuestos por Cohen: $\sin$ efecto $<.20$, efecto leve $=.21$ a.49, efecto moderado $=.50$ a $.79 \mathrm{y}$ efecto grande $>$. 80. En el cuadro 1, se integran tanto los resultados de las pruebas PSHN y ME.

\section{(Cuadro 1)}

Los resultados obtenidos en las medidas indirectas de las FE mediante la ME, confirman las diferencias observadas con base en la PSHN ( $U$ de Mann-Witney). Estas diferencias observadas en los factores de la escala BRIEF fluctuaron

Cuadro 1. Comparación de las FE entre los grupos

\begin{tabular}{|c|c|c|c|c|c|c|c|}
\hline \multirow[b]{2}{*}{ BRIEF } & \multicolumn{2}{|c|}{ TEA } & \multicolumn{2}{|c|}{ Control } & \multirow[b]{2}{*}{ U } & \multirow[b]{2}{*}{ p } & \multirow[b]{2}{*}{ d } \\
\hline & M & DE & M & DE & & & \\
\hline Inhibición & 20.86 & 3.85 & 12.57 & 3.82 & 3.5 & .006 & 2.16 \\
\hline Flexibilidad & 14.86 & 1.77 & 11.14 & 2.48 & 4.5 & .010 & 1.73 \\
\hline Control emocional & 20.71 & 4.82 & 14.00 & 3.83 & 6.5 & .020 & 1.54 \\
\hline BRI & 56.43 & 9.81 & 37.71 & 9.39 & 5.5 & .014 & 1.95 \\
\hline Iniciación & 15.71 & 3.30 & 11.43 & 2.57 & 6 & .016 & 1.45 \\
\hline Memoria de trabajo & 21.57 & 3.60 & 14.14 & 4.45 & 5.5 & .014 & 1.84 \\
\hline Planeación & 26.43 & 4.20 & 16.00 & 6.19 & 5 & .012 & 1.97 \\
\hline Organización de materiales & 13.86 & 2.61 & 10.86 & 2.91 & 9.5 & .052 & 1.09 \\
\hline Monitoreo & 18.29 & 4.31 & 12.14 & 3.13 & 6 & .017 & 1.63 \\
\hline MI & 95.86 & 14.28 & 64.57 & 17.76 & 5 & .012 & 1.94 \\
\hline GEC & 152.29 & 20.11 & 102.29 & 26.74 & 5.5 & .014 & 2.11 \\
\hline \multicolumn{8}{|l|}{ ENFEN } \\
\hline Fluidez fonológica & 5.28 & 1.97 & 8.00 & 3.91 & 12.5 & .121 & -0.88 \\
\hline Fluidez semántica & 10.85 & 5.84 & 14.71 & 2.13 & 13.5 & .159 & -0.88 \\
\hline Sendero gris & 12.70 & 4.72 & 19.06 & 5.43 & 12 & .110 & -1.25 \\
\hline Sendero a color & 5.74 & 2.51 & 8.66 & 2.07 & 8 & .035 & -1.26 \\
\hline Anillas & 420.57 & 160.7 & 335.00 & 67.53 & 17 & .338 & 0.69 \\
\hline Interferencia & 42.02 & 19.18 & 55.19 & 16.06 & 18 & .406 & -0.74 \\
\hline
\end{tabular}


entre $d=1.09$ (dificultades en la organización de materiales) y 2.16 (dificultades de inhibición), a favor del grupo con TEA. Por su parte en las $\mathrm{MD}$, solo la subprueba sendero a color obtuvo diferencias tanto en PSHN (.035) como en la $M E(d=-1.26)$.

El reanálisis de nuestros datos, con la inclusión de la $\mathrm{ME}$, confirman la conclusión número dos hecha en nuestro documento: "Los niños con TEA son evaluados con más dificultades de inhibición, flexibilidad, iniciación, planeación/ organización, monitoreo, memoria de trabajo y control emocional." Por otra parte, se encontraron diferencias en la mayoría de las MD de las FE entre niños con y sin TEA de acuerdo con la ME $(d>.80)$, lo que modifica conclusión numerada con el cuatro "No se encontraron diferencias en la mayoría de las funciones ejecutivas evaluadas directamente entre niños con TEA y neurotípi$\cos ^{\prime \prime}$, ya que esta conclusión se basó únicamente en la PSHN.

Las diferencias entre las medidas directas e indirectas basadas en nuestro estudio en la ME apoyan la hipótesis de un perfil de FE en niños con TEA, distinto a los de desarrollo neurotípico.

Determinar el perfil neuropsicológico de las FE de niños con TEA aún representa un reto científico. No obstante, nuestro trabajo en el marco de sus limitaciones puede ser considerado como un estudio piloto y de factibilidad para la medición directa e indirecta de las funciones ejecutivas en los niños con TEA.

\section{REFERENCIAS}

1. Leung, RC, Zakzanis KK. Brief report: cognitive flexibility in autism spectrum disorders: a quantitative review. Journal of autism and developmental disorders. 2014; 44 (10): 2628-2645.

2. Rosenthal M, Wallace G, Lawson R et al. Impairments in Real World Executive Function Increase from Childhood to Adolescence in Autism Spectrum Disorders. Neuropsychology. 2013; 27 (1): 13-18.

3. Gioia GA, Isquith OK, Kenworthy L, Barton RM. Profiles of everyday executive function in acquired and developmental disorders.Child Neuropsychol. 2002; 8 (2): 121-137.

4. Weston L, Hodgekins J, Langdon, PE. Effectiveness of cognitive behavioural therapy with people who have autistic spectrum disorders: A systematic review and meta-analysis. Clin Psychol Rev. 2016; 49: 41-54.

5. Merchán-Naranjo J, Boada L, Rey-Mejías A, Mayoral M, Llorente C, Arango C, Parellada M. La función ejecutiva está alterada en los trastornos del espectro autista, pero está no correlaciona con la inteligencia. Revista de Psiquiatría y Salud Mental. 2016; 9(1): 39-50.

6. Pérez-Pichardo MF, Ruz-Sahrur A, Barrera-Morales K, MooEstrella J. Medidas directas e indirectas de las funciones ejecutivas en niños con trastorno de espectro autista. Acta Pediatr Mex. 2018; 39(1):13-22.

7. Shapiro SS, Wilk MB. An Analysis of Variance Test for Normality (Complete Samples). Biometrika. 1965; 52 (4): 591-611.

8. Rivas-Ruiz R, Moreno-Palacios J, Talavera J. Diferencias de medianas con U de Mann Witney. Rev Med Inst Mex Seguro Soc. 2013; 51 (4): 414-9.

9. Cohen J. A power primer. Psychol Bull. 1992; 112: 155-9.

Pérez Pichardo MF, Moo Estrella JA

Laboratorio de sueño

Facultad de Psicología de la Universidad Autónoma de Yucatán.

Campus Ciencias Sociales, Económico-Administrativas y Humanidades.

Kilómetro 1 Carretera Mérida Tízimin-Cholul jmestre@correo.uady.mx 